First publ. in: Science 287 (2000), 5453, pp. 625-627

\title{
Single-File Diffusion of Colloids in One-Dimensional Channels
}

\author{
Q.-H. Wei, ${ }^{*} \dagger$ C. Bechinger, ${ }^{*}$ P. Leiderer
}

\begin{abstract}
Single-file diffusion, prevalent in many processes, refers to the restricted motion of interacting particles in narrow micropores with the mutual passage excluded. A single-filing system was developed by confining colloidal spheres in one-dimensional circular channels of micrometer scale. Optical video microscopy study shows evidence that the particle self-diffusion is non-Fickian for long periods of time. In particular, the distribution of particle displacement is a Gaussian function.
\end{abstract}

Single-file diffusion (SFD) occurs when the individual pores of the medium are so narrow that the particles are unable to pass each other $(1,2)$. The sequence of particles remains unchanged over time, and thus, the basic principle of diffusion as a physical mixing process comes into question. The concept of SFD was originally introduced more than 40 years ago in biophysics to account for the transport of water and ions through molecular-sized channels in membranes (3); since then, in addition to biological systems $(4,5)$, SFD is also discussed in the context of interaction of Markov chains in statistics (6), the transportation of adsorbate molecules through zeolites (2), and charge-carrier migration in one-dimensional (1D) polymer and superionic conductors (7). Furthermore, SFD is also related to surface growth phenomena through some mapping (8).

As the mutual passage of particles is prohibited in single-filing (SF) systems, the movements of individual particles are correlated, even at long time periods, because the displacement of a given particle over a long distance necessitates the motion of many other particles in the same direction. This correlation is reflected in the long-time behavior of the mean-square displacement (MSD), which has been predicted for an infinite system to be $(6,7,9-11)$

$$
\left\langle\Delta x^{2}\right\rangle=2 F \sqrt{t}
$$

Faculty of Physics, University of Konstanz, Postfach M676, D-78457 Konstanz, Germany.

*To whom correspondence should be addressed. E-mail: qwei+@pitt.edu (Q.-H.W.) and clemens. bechinger@uni-konstanz.de (C.B.)

$\dagger$ Present address: Department of Physics and Astronomy, University of Pittsburgh, Pittsburgh, PA 15260 USA. where $F$ is the SF mobility and $t$ is time. Accordingly, SFD processes, in contrast to 2D and 3D self-diffusion seen in colloidal systems (12), cannot be described by a diffusion coefficient; that is, the SFD does not obey Fick's laws.

Experimental evidence confirming nonFickian behavior was unavailable for a long time because of the lack of ideal experimentally accessible SF systems. Recently, measurements of SFD became feasible in artificial crystalline zeolites. Adsorbate molecules, like methane or $\mathrm{CF}_{4}$ with diameters of 3.8 and $4.7 \AA$, respectively, confined in $\mathrm{AlPO}_{4}-5$ zeolite with a pore size of $7.3 \AA$, are considered to be good realizations of SF systems. Although some experimental evidence for the occurrence of SFD was found by pulsed field gradient nuclear magnetic resonance study $(13,14)$, some results from different groups and experimental methods are still in contradiction, even for the same system $(15,16)$, as indicated by Hahn and Kärger (17), who suggest and explore several possible reasons. Other effects, such as attractive particle interaction (18), the possible existence of correlations between particles of neighboring pores (19), have also been shown to play a vital role in the mechanism and rates of intracrystalline diffusion. Because of the shortage of structural information on the atomic level, the mechanism of molecular diffusion in zeolites, however, is still under debate.

We created a well-defined SFD model system by confining paramagnetic colloidal spheres of several micrometers in a set of circular trenches fabricated by photolithography. The channels are well-characterized, and the particle-particle interaction can be precisely adjusted by an external magnetic field. Moreover, because the time and length scales in such a colloidal system are easily accessed 
Fig. 1. (A) Scanning electron microscope image of the 1D trenches fabricated on the photoresist polymer film by photolithography. (B) Optical microscope image of three concentric circular channels with colloidal particles confined in them (the small black objects inside the channels). After the cell was assembled, the col-
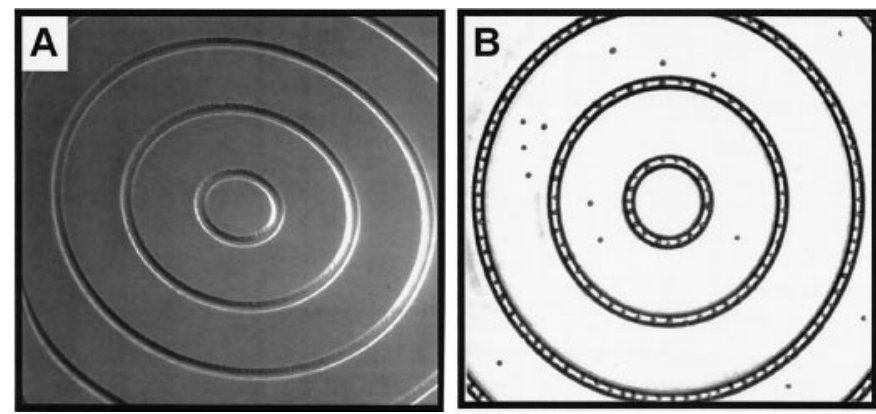

loidal particles sedimented to the bottom plate under the action of gravitation and were trapped in the microchannels (promoted by slightly tilting the substrate). With a particle density of $1.20 \mathrm{~g} \mathrm{~cm}^{-3}$, the gravitation potential well of the $5-\mu \mathrm{m}$-deep channel is $\sim 400 k_{\mathrm{B}} T$ in depth. After most of the particles were trapped in the channels, the sample was carefully adjusted to horizontal before the experiments. The dynamics of colloidal particles was monitored with a home-built inverted transmission optical microscope system that was connected to a charge-coupled device camera and a computer.

Fig. 2. (A) Typical trajectories for eight neighboring particles in the largest channe in Fig. 1A. The instantaneous particle coordinates were extracted from digitized pictures with an imageprocessing algorithm and saved in a computer for later analysis. From those data, we obtained the parti-

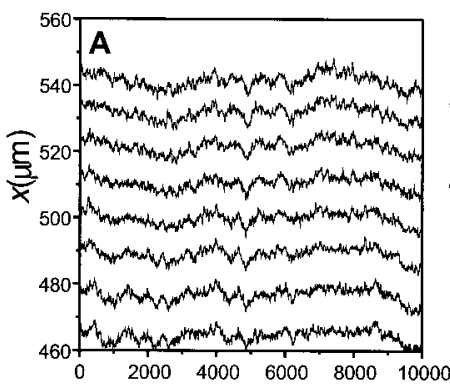

$t$ (s)

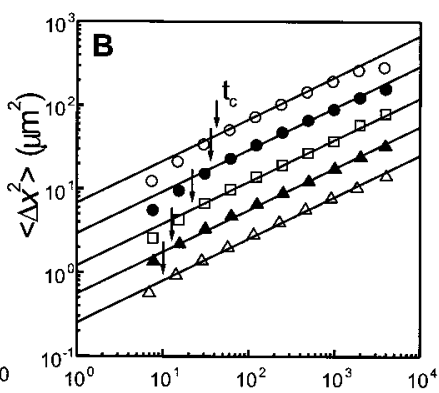

$t$ (s)

cle trajectories. The system was equilibrated for at least 4 hours before each measurement. To obtain the long-time behavior, we recorded the coordinates of colloidal particles for $\sim 8$ hours, with a time interval of $\sim 8 \mathrm{~s}$ between two adjacent pictures. (B) Log-log plot of the measured particle MSDs versus the observation time for five different particle interaction strengths $\Gamma: 0.66$, open circles; 1.1 , solid circles; 2.34 , open squares; 4.03 , solid triangles; and 7.42 , open triangles. The data points have been shifted upward by $\ln 2$ for clarity, and the solid lines are best fit with Eq. 1 with the mobility $F$ as an adjustable parameter.

Fig. 3. (A) The particle displacement distribution function $p(x, t)$ for $\Gamma=4$ at different times $t: 77 \mathrm{~s}$, circles; $385 \mathrm{~s}$, triangles; $770 \mathrm{~s}$, squares; and $3850 \mathrm{~s}$, inverted triangles. (B) The distribution function $p(x, t)$ of $(\mathrm{A})$ replotted by scaling $P(x$, $t) \rightarrow t^{1 / 4} p(x, t), x \rightarrow$ $x / t^{1 / 4}$. The solid curve is a Gaussian fit with
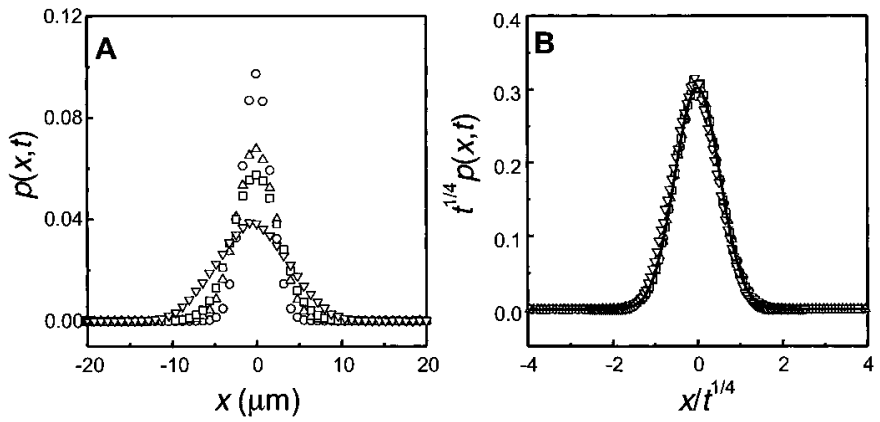

Eq. 2, with the fitting parameter $F=0.14 \mu \mathrm{m} / \mathrm{s}^{1 / 2}$.

with video microscopy, the trajectories of individual particles can be followed over long periods of time. We unambiguously observed the non-Fickian behavior of SFD and confirm the theoretically predicted Gaussian distribution of particle displacements.

The sample cell was composed of two optical flats separated by an $\mathrm{O}$ ring of $0.5-\mathrm{mm}$ thickness. The bottom plate was first coated with a thin layer of poly(methyl methacrylate) to prevent colloidal particles from sticking to the glass surface. On top of this film, a 5- $\mu \mathrm{m}-$ thick layer of a transparent photoresist was deposited. Afterward, a set of concentric circular channels ( $7 \mu \mathrm{m}$ in width and 33 to $1608 \mu \mathrm{m}$ in diameter) was etched into the photoresist by means of photolithography (Fig. 1A). The distance between adjacent channels, $63 \mu \mathrm{m}$, is much larger than the mean particle separation $(11 \mu \mathrm{m})$, which rules out correlation between particles in neighboring channels.

We used an aqueous suspension of paramagnetic polystyrene colloids with a diameter of 3.6 $\mu \mathrm{m}$ (M-350 Dyno AS, Lillestroem, Nor- way). The particles were doped with $\mathrm{Fe}_{2} \mathrm{O}_{3}$ clusters and are paramagnetic. Thus, when an external magnetic field is applied perpendicular to the sample plane, a magnetic dipole moment $M$ is induced in the colloids (which is proportional to the weak field strengths $B$ that we used) that gives rise to a repulsive pair interaction potential of the form $V(r)=\left(\mu_{0}\right)$ $4 \pi) M^{2}(B) r^{-3}$, where $r$ is the particle distance and $\mu_{0}$ is the vacuum permeability. In order to characterize the interaction strength between particles, we introduce the quantity $\Gamma=\beta\left(\mu_{0}\right)$ $4 \pi) \chi_{\text {eff }}^{2} B^{2} R^{-3}$, which is the mean interaction energy normalized by the thermal energy $1 / \beta=$ $k_{\mathrm{B}} T$, where $k_{\mathrm{B}}$ is the Boltzmann constant and $T$ is the temperature. Here, $R$ is the mean particle distance, and $\chi_{\text {eff }}$ is the magnetic susceptibility of the particles, which was determined to be $2.2 \times 10^{-12} \mathrm{~A} \mathrm{~m}^{2} \mathrm{~T}^{-1}$ (20). The particle-wall interaction can be considered as a hard spherewall interaction to a good approximation.

A typical real-space configuration of the particles at $\Gamma=4$ is shown in Fig. 1B. Each channel appears as two closely spaced concentric rings because of the diffraction of the light at its walls into the photoresist. The particles are the dark objects within the channels. Most of the particles are trapped in the channels, whereas only a few reside on the elevated areas and do not influence the diffusion behavior of particles in the channels. The channels are narrow enough to meet the SF condition and prohibit the mutual passage of particles.

In Fig. 2A, we plotted typical trajectories of eight neighboring particles obtained at $\Gamma=$ 4 from the largest channel in Fig. 1B. The particle position $x$ corresponds to their angular coordinate multiplied by the channel radius. As can be seen, the trajectories never cross during the measuring time, which indicates that the SF condition is satisfied. The correlation of particle positions for long periods of time, as a characteristic of SFD, can also be seen from Fig. 2A.

The results of MSD in a log-log plot for five different magnetic field strengths $(\Gamma=0.66$, $1.10,2.34,4.03$, and 7.42) are shown in Fig. 2B. For the calculation of MSD, we averaged over both the time $t$ and all of the particles in the channel, or $\left\langle\Delta x^{2}\right\rangle=\Sigma_{i}\left\langle\left[x_{i}\left(t+t^{\prime}\right)-x_{i}\left(t^{\prime}\right)\right]^{2}\right\rangle_{t^{\prime}} / N$, where $N$ is the total number of particles and $i$ is the particle index. The solid lines in Fig. 2B correspond to the best fits according to Eq. 1 with the mobility $F$ as the only adjustable parameter. The predicted $t^{1 / 2}$ behavior was seen over more than two decades of time. The small deviations from the solid lines at small time scales correspond to the crossover between the long-time and short-time diffusion of the particles. The crossover time $t_{\mathrm{c}}$ (arrows in Fig. 2B) is significantly shifted to larger values when the magnetic dipole repulsion between the particles decreases.

We also calculated the distribution function of displacements $p(x, t)$, which is defined 


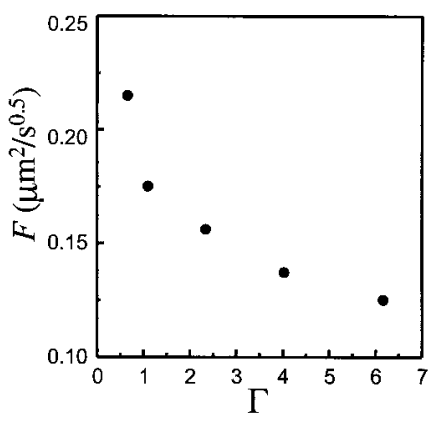

Fig. 4. The single-file particle mobility obtained from the fittings shown in Fig. $2 \mathrm{~B}$ as a function of the normalized particle interaction strength $\Gamma$.

as the conditional probability of finding a particle at position $x$ after time $t$ with the particle located for $t=0$ at $x=0$. In Fig. 3A, we show the result of $p(x, t)$ for $\Gamma=4$ at four different times, which are all greater than $t_{\mathrm{c}}$. Self-diffusion of particles causes $p(x, t)$ to broaden with time.

Despite the simplicity of the physical situation describing SF conditions, theoretical treatment remains a highly sophisticated task. Analytical results are only obtained for long time limits for hard rods hopping in an infinite 1D lattice (called a 1D exclusion model). It has been predicted that $p(x, t)$ follows $(6$, 10,14 )

$$
p(x, t)=\frac{1}{\sqrt{4 \pi F} t^{1 / 4}} \exp \left(-x^{2} / 4 F t^{1 / 2}\right)
$$

This form, however, is suggested to remain valid under more general conditions whenever the SF effect is important.

To compare our data with Eq. 2, we replotted the data of Fig. 3A in Fig. 3B; all of the data points collapse to a master curve after a rescaling of the axis. In addition, a Gaussian function fit (Fig. 3B, solid curve) shows good agreement with the data. From the only adjustable parameter of the Gaussian fit, the SF mobility can be derived, which is in agreement with the value obtained from the MSD data. This observation is also true for the $p(x, t)$ for the other magnetic fields. It should be emphasized that Eq. 2 has not been directly observed in experiments before.

The 1D exclusion model predicts that $F$ decreases with the particle density $\rho$ according to $F \propto(1-\rho) / \rho$. In our system, however, it is more convenient to change the particle interaction strength $\Gamma$ while keeping the particle density constant. This change is equivalent to changing the particle density because an increase in $\Gamma$ results in an increase of collision rates between particles or, equivalently, an increase in particle density. The measured mobility (Fig. 4) decreases with the increase of particle interaction energy, which is qualitatively in agreement with the theoretical prediction.
However, unlike the hard-rod interaction in the theoretical exclusion model, we have a longrange pair interaction, and the hydrodynamic interactions caused by the particles moving in the surrounding fluid also play an importan role. Therefore, detailed comparison with theory should take these two aspects into account.

\section{References and Notes}

1. E. J. Harris, Transport and Accumulation in Biological Systems (Butterworths Scientific, London, 1960); B. Alberts et al., Molecular Biology of the Cell (Garland New York, 1994)

2. J. Kärger and D. M. Ruthven, Diffusion in Zeolites and Other Microporous Solids (Wiley, New York, 1992); N. Y. Chen, T. F. Degnan, C. M. Smith, Molecular Transport and Reaction in Zeolites ( $\mathrm{VCH}$, New York, 1994).

3. A. L. Hodgkin and R. D. Kenes, J. Physiol. (London) 128, 61 (1955)

4. E. J. A. Lea, J. Theor. Biol. 5, 102 (1963); H. Rickert, Z. Phys. Chem. Neue. Folge. 43, 129 (1964); D. G. Levitt, Biochim. Biophys. Acta 373, 115 (1974).

5. P. A. Rosenberg and A. Finkelstein, J. Gen. Physiol. 72 341 (1978); S. Draber, R. Schultze, U.-P. Hansen, J. Membr. Biol. 123, 183 (1991); J. A. Hernandez and J. Fischbarg, J. Gen. Physiol. 99, 645 (1992); T. Chou and D. Lohse, Phys. Rev. Lett. 82, 3552 (1999).

6. R. Arratia, Ann. Probab. 11, 362 (1983).

7. P. M. Richards, Phys. Rev. B 16, 1393 (1977); P. A Fedders, Phys. Rev. B 17, 40 (1978).
8. T. Halpin-Healy and Y. C. Zhang, Phys. Rep. 254, 215 (1995).

9. S. Alexander and P. Pincus, Phys. Rev. B 18, 2011 (1978).

10. H. van Beijeren, K. W. Kehr, R. Kutner, Phys. Rev. B 28, 5711 (1983).

11. J. Kärger, Phys. Rev. A 45, 4173 (1992); Phys. Rev. E 47, 1427 (1993); K. Hahn and J. Kärger, J. Phys. A Math. Gen. 28, 3061 (1995).

12. G. Nägle, Phys. Rep. 272, 215 (1996); X. Qiu, H. D. Ou-Yang, P. M. Chaikin, J. Phys. (Paris) 49, 1043 (1988); A. van Blaaderen, J. Peetermans, G. Maret, J. K. G. Dhont, J. Chem. Phys. 96, 4591 (1992).

13. V. Gupta et al., Chem. Phys. Lett. 247, 596 (1995)

14. V. Kukla et al., Science 272, 702 (1996); K. Hahn, J. Kärger, V. Kukla, Phys. Rev. Lett. 76, 2762 (1996).

15. S. S. Nivarthi, A. V. McCormick, H. T. Davis, Chem. Phys. Lett. 229, 197 (1994).

16. H. Jobic et al., J. Phys. Chem. B 101, 5834 (1997).

17. K. Hahn and J. Kärger, J. Phys. Chem. B 102, 5766 (1998).

18. D. S. Sholl and K. A. Fichthorn, Phys. Rev. Lett. 79, 3569 (1997).

19. R. Radhakrishnan and K. Gubbins, Phys. Rev. Lett. 79, 2847 (1997).

20. K. Zahn, thesis, University of Strasbourg, Strasbourg France (1997)

21. We thank K. Zahn for giving us the paramagnetic colloidal particles. The work is supported by Deutsche Forschungsgemeinschaft through grant SFB513.

20 September 1999; accepted 8 December 1999 